\title{
Does the BMJ have a particular ideology to pursue in assisted dying?
}

Owing to an error during the processing of this letter, the email address of the author, Adrian M Houghton, is incorrect in the print version (and its associated section pdf) (BMJ

2012;344:e731, doi:10.1136/bmj.e731). The correct email address is meh125@doctors.org.uk. 